\title{
From Molecules to Solids: A vdW-DF-C09 Case Study of the Mercury Dihalides
}

\author{
Valentino R. Cooper ${ }^{\mathrm{a}, *}$, Jaron T. Krogel ${ }^{\mathrm{a}}$, Kelling J. Donald ${ }^{\mathrm{b}, *}$
}

a Materials Science and Technology Division, Oak Ridge National Laboratory, Oak Ridge, Tennessee, 37830, United States

${ }^{b}$ Department of Chemistry, Gottwald Center for the Sciences, University of Richmond, Richmond, VA 23173, United States

\section{Supporting Information}

*Corresponding authors. V. R. Cooper Tel.: 1-865-574-5164. E-mail: coopervr@ornl.gov; ORCID: 00000001-6714-4410. K. J. Donald Tel.: 1-804-484-1628. E-mail: kdonald@richmond.edu ORCID: 00000001-9032-4225 


\begin{tabular}{|l|l|c|}
\hline Figure S1 & $\begin{array}{l}\text { Caption } \\
\text { Graphical comparison of vdW-DF-C09 condensation energies vs PBE- } \\
\text { D3 values. A strong linear correlation is observed with a 0.40 eV shift in } \\
\text { the binding energies. This may be indicative of some vdW-DF-C09 } \\
\text { overbinding and underbinding by the } \mathrm{PBE}-\mathrm{D} 3 \text { approach. }\end{array}$ & S3 \\
\hline Figure S2 & $\begin{array}{l}\text { Condensation energy for } \mathrm{HgCl}_{2}, \mathrm{HgBr}_{2} \text { and } \mathrm{HgI} \text { for the four possible } \\
\text { crystal structures considered obtained from } \mathrm{PBE}-\mathrm{D} 3 \text { calculations. In all } \\
\text { cases, the reference energy is the isolated } \mathrm{Hg} X_{2} \text { molecule. }\end{array}$ & S4 \\
\hline File S1 & NEXUS Submission script for job management. & S5-S8 \\
\hline
\end{tabular}




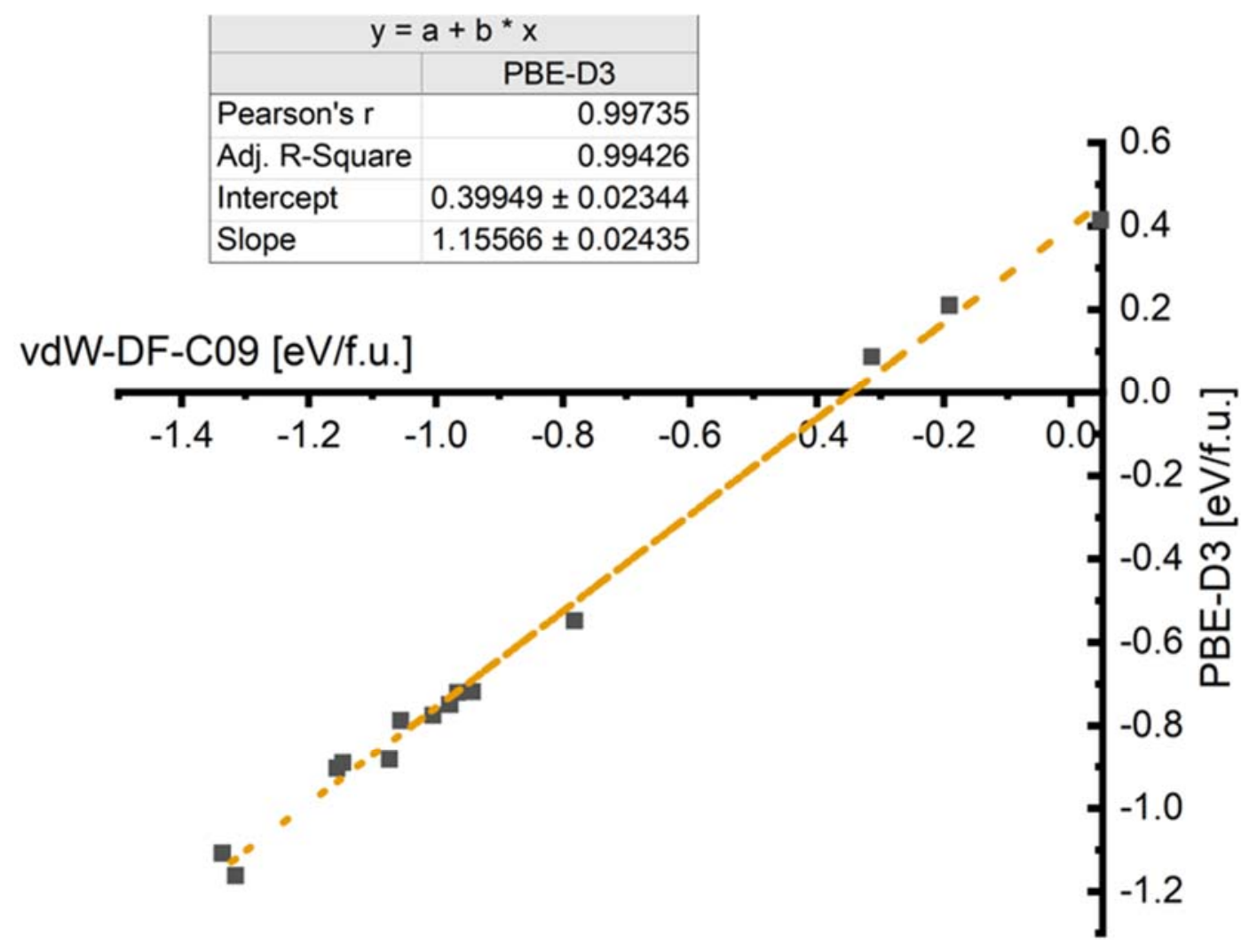

\section{$\Delta E_{\mathrm{vdW}-\mathrm{DF}-\mathrm{CO9}}=0.400+1.156 \Delta E_{\mathrm{PBE}-\mathrm{D} 3}$}

Figure S1: Graphical comparison of vdW-DF-C09 condensation energies vs PBE-D3 values. A strong linear correlation is observed with a $0.40 \mathrm{eV}$ shift in the binding energies. This may be indicative of some vdW-DF-C09 overbinding and underbinding by the PBE-D3 approach. 


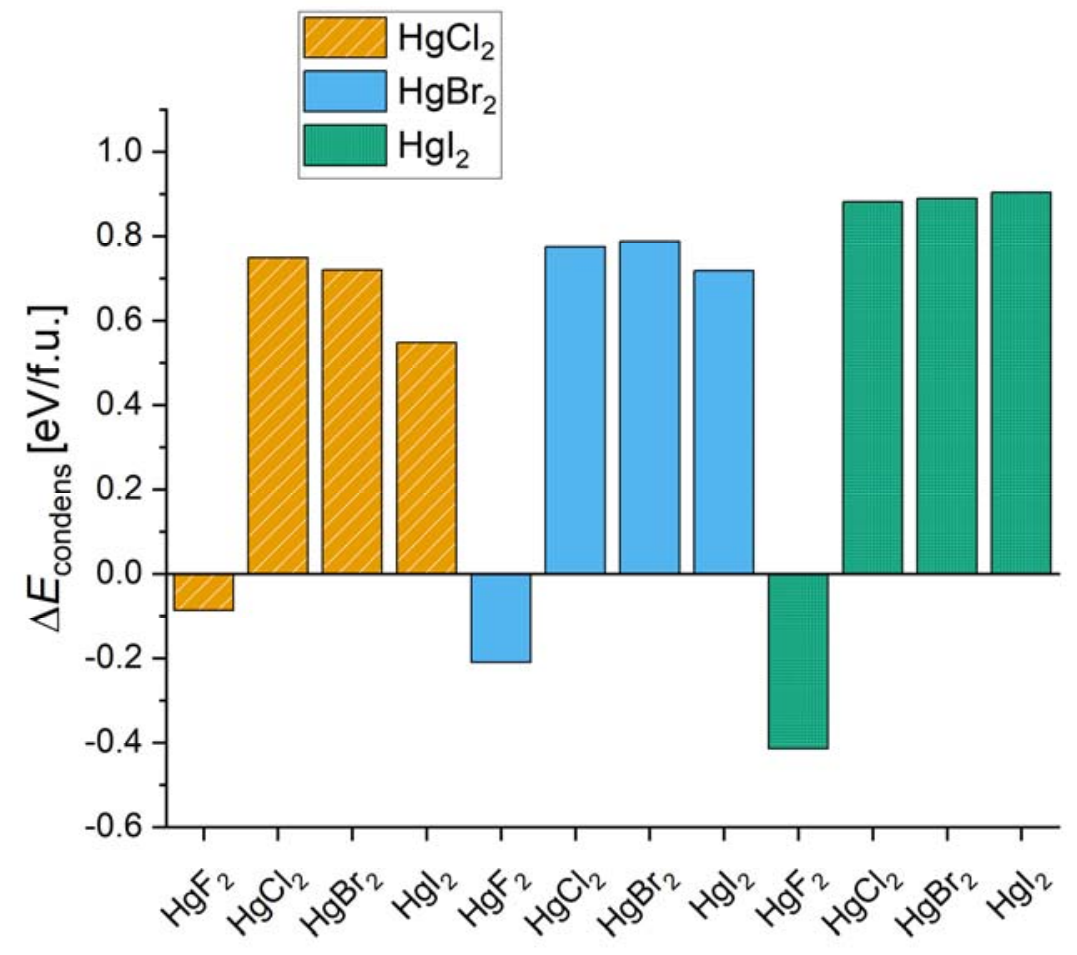

Figure S2: Condensation energy for $\mathrm{HgCl}_{2}, \mathrm{HgBr}_{2}$ and $\mathrm{HgI}_{2}$ for the four possible crystal structures considered obtained from PBE-D3 calculations. In all cases, the reference energy is the isolated $\operatorname{Hg} X_{2}$ molecule. 
File S1: NEXUS Submission script for job management.

The NEXUS workflow management code can be found at: $\underline{\text { https://qmcpack.org/nexus }}^{1}$.

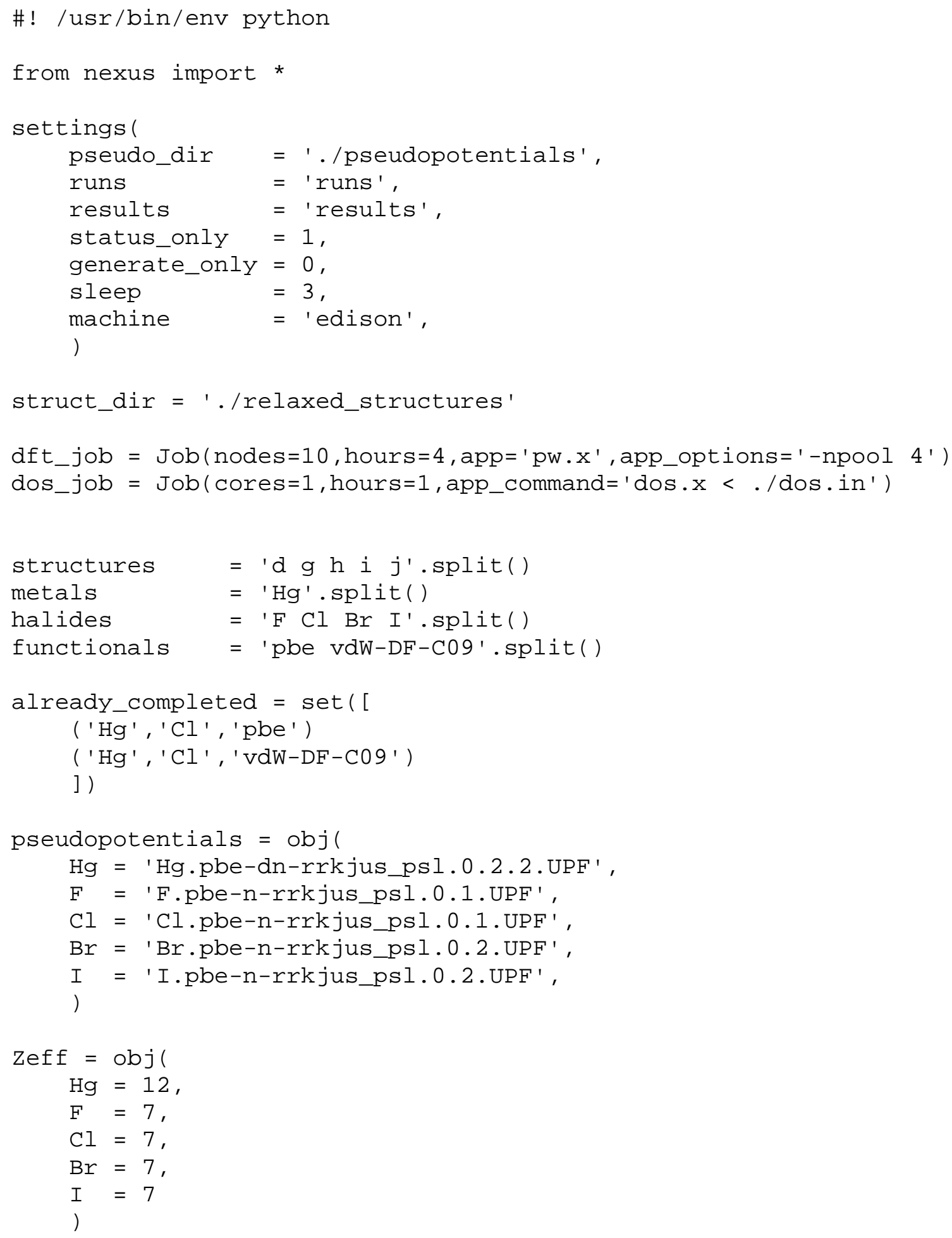




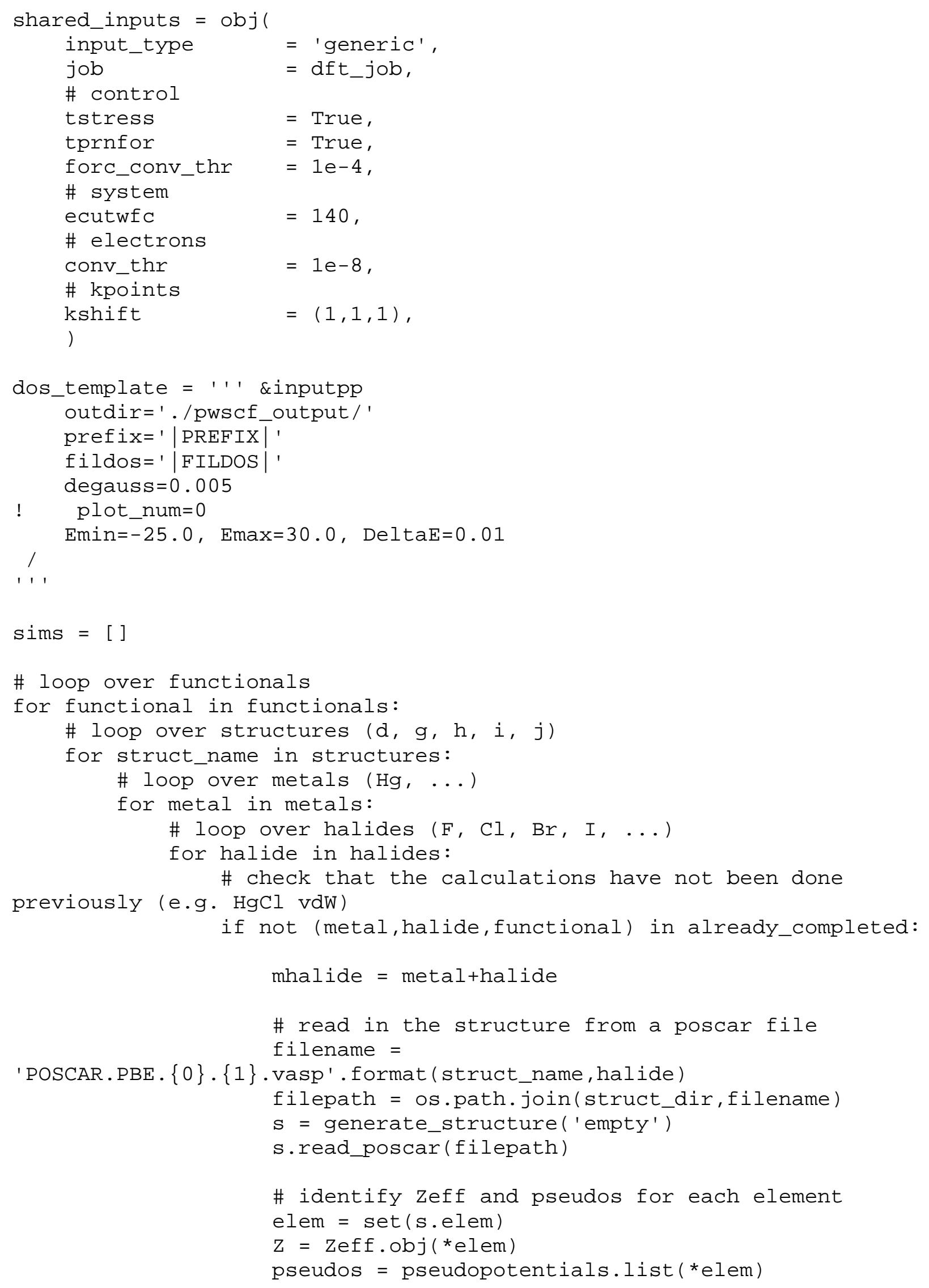


info)

\# create physical system (ion pos + electronic

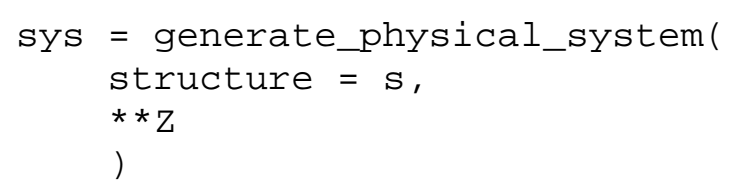

$'\{0\} .\{1\} .\{2\}$ '. format (functional, struct_name, mhalide)

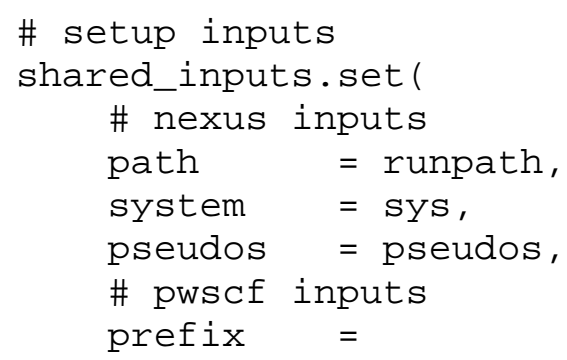

' $\{0\}_{-}\{1\}$.dos' . format (mhalide, functional),

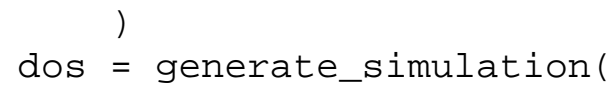




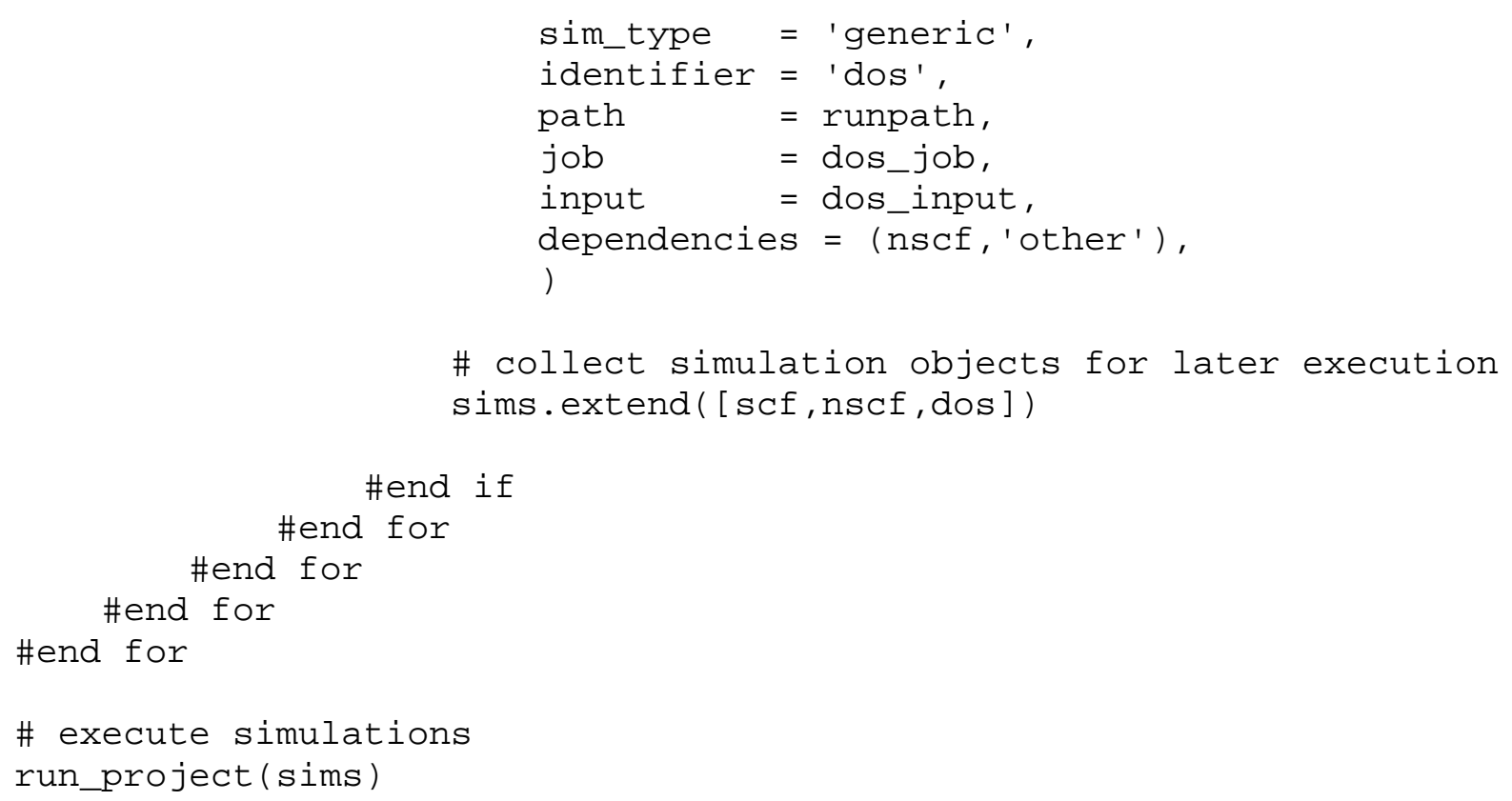

\section{Reference:}

1. Krogel, J., Nexus: A modular workflow management system for quantum simulation codes. Comput. Phys. Commun. 2016, 198, 154-168. 